\title{
Assumptions, conceptualizations, and the search for the functions of the brain*
}

\author{
WILLIAM G. WEBSTER \\ Carleton University: Ottawa. Ontario, Canada
}

\begin{abstract}
The conditions under which methods of direct neurological manipulation may be of utility in the study of brain-behavior relations and of cerebral localization of function are discussed, with attention being directed in particular to the conflict of positions represented by R. L. Gregory and L. Weiskrantz. The argument is made that such methods are potentially useful only if the nervous system or particular portions of the nervous system can be conceptualized in terms of being a parallel, rather than a serial information processing system. It is further argued that inferences made on the basis of results generated with such methods (and indeed any method) are dependent upon the assumptions and conceptualizations concerning brain function held by the $\mathrm{E}$.
\end{abstract}

Much of the history and the contemporary concern of physiological psychology focuses upon the related issues of the identification of the functions of the brain and cerebral localization of those functions. These issues of brain-behavior relations are of ten identified with cortical functioning, perhaps in part because of the controversy which surrounded Lashley's concepts of equipotentiality and mass action, but the history of attempts to identify the functions of the subcortical neuroanatomical structures is certainly as real. It is reflected, for instance, in discussions of homeostatic feeding and satiety centers, centers for the regulation of water balance and body temperature, and neural substrates of maternal, sexual, and aggressive behaviors. It is reflected in such titles of contemporary literature of physiological psychology as "Functions of the amygdala" (Goddard, 1964), "Hippocampus and internal inhibition" (Kimble, 1968), "Septum and behavior: A review" (Fried, 1972), Functions of the corpus callosum (Ettlinger, 1965), or "Approach-avoidance dissociation in rat brain" (Olds, 1960), to name but a few, as well as in chapter and section headings of most contemporary textbooks of physiological psychology.

There are certain problems and issues of a conceptual nature which are implicit in attempts to specify brain function using the classical neuropsychological experimental method involving direct manipulation of the brain. These issues, which have profound implications for research methodology and data interpretation, can be seen in rather sharp focus when viewed against a historical backdrop of the development of this experimental method and of the parallel development of thought during the 19th century concerning cerebral localization of function.

\section{A HISTORICAL OVERVIEW}

There are several very adequate accounts of the

*Preparation of this manuscript was supported in part by Grant APA-0399 from the National Research Council of Canada. modern history of cerebral localization of function (e.g., Boring, 1950; Walker, 1957; and especially, Young, 1968, 1970), and consequently only a few points concerning the basic logic of the experimental method as applied to the study of brain-behavior relations need be mentioned and commented upon.

The development of this experimental method is usually credited to Pierre Flourens (1794-1867) and was in part a reaction to the phrenological approach of Franz Joseph Gall (1758-1828). The conceptual and methodological framework of Gall's approach to the understanding of individual differences in behavior has been very elegantly and succinctly sketched by Young $(1970$, p. 36) as follows:

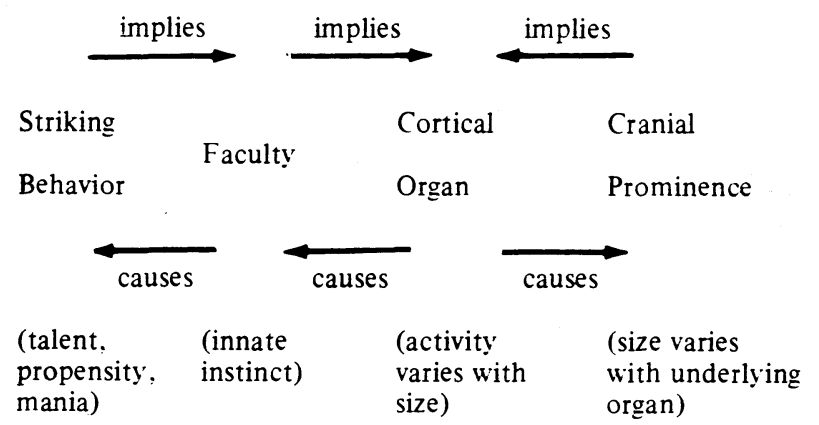

For Gall, individual differences in behavior were to be accounted for in terms of innate or inborn faculties. These faculties of the mind were seen as being localized within the brain, and for each distinct faculty there was presumed to be a separate and distinct cerebral organ. It was the size of the cerebral organ that determined the strength of the associated faculty, and the relative strengths of the faculties were reflected in the form of talents or other outstanding behavioral attributes. Furthermore, the size of each cerebral organ was reflected in the size of the overlying cranium.

Within this framework, the study of individual differences had then two facets: first, the identification 
of the faculties of the raind, which were equated with the functions of the brain; and second, the localization of function in various parts or organs of the brain. It was in the first facet that Gall was, in fact, more concerned and interested, and he argued that the functions of the brain were ones of biological significance, specifically rejecting the view that these necessarily included the categories, derived from philosophy, of perception, attention, judgment, memory, imagination, and so on. Furthermore, he argued that they could be discovered only by observation and empirical study, in much the same way as could the functions of any part of the body be discovered. Basically, his methodology involved correlating the presence of cranial prominences (from which he inferred the size of the underlying cerebrum) of living individuals, skulls and head casts, with the manifestation of striking talents or propensities (from which he inferred the existence and relative strengths of faculties). From this, he ultimately concluded that there were some 37 separate, distinct, and highly localized human faculties.

It was against this emphasis on a high degree of localization of function that Pierre Flourens (1794-1867) argued on the basis of his experimental method of brain ablation. For Flourens, the problem of determining and localizing brain function was simply one of making inferences about faculties based on changes in behavior which followed the removal of cerebral tissue. The logic of this experimental method was deceptively simple: remove a part of the brain, observe what the organism ceases to do, and infer the normal function of the removed part. His most notable success within this orientation was in the study of the cerebellum of pigeons. The removal of successive slices of cerebellar tissue led to an increasing loss of motor control, and from this Flourens made the reasonable inference that the cerebellum was concerned with motor coordination and not with reproduction, as Gall had maintained.

At this point, it is instructive to contrast Flourens's observations of the behavioral changes which followed the removal of the cerebrum of a hen with the inferences he made on the basis of these:

"I let this hen starve several times for as long as three days. Then I brought nourishment under her nose, I put her beak into grain. I put grain into her beak. I plunged her beak into water, I placed her on a shock of corn. She did not smell, she did not eat anything. she did not drink anything, she remained immobile on the shock of corn and she would certainly have died of hunger if I had not returned to the old process of making her eat by herself.

"Twenty times, in lieu of grain. I put sand into her beak: and she ate this as she would have eaten grain.

"Finally, when this hen encounters an obstacle in her path, she throws herself against it. and this collision stops her and disturbs her, but to collide with an object is not the same as to touch it .... She is collided with and she collides but she does not touch [Flourens. 1842. pp. 90-91: translated by Young. 1970].."
Flourens made the intuitively reasonable inference that the bird had lost its senses, but then he proceeded to make the following inferences about the functions of the cerebrum:

"An animal which really touches a body, judges it; an animal which does rot judge anymore therefore does not touch anymore.

"Animals deprived of their cerebral lobes have, therefore, neither perception, nor judgment, nor memory, nor will: because there is no volition when there is no judgment; no judgment when there is no memory; no memory when there is no perception. The cerebral lobes are therefore the exclusive seat of all the perceptions and of all the intellectual faculties [Flourens, 1842, pp.96-97; translated by Young. 1970]."

It is evident from this quotation that Flourens not only rejected Gall's methodology, but also his ideas on the nature of the functions of the brain, and echoed the classical faculties of perception, will, volition, and memory. Furthermore, the quotation indicates the basis of his arguments against the doctrine of cerebral localization: his ablations did not result in changes in one behavior and not others, but produced graded deficits in all the behaviors, which he noted. However, it is important to appreciate that even if there were localization of function in the various parts of the cerebrum, an ablation method as neuroanatomically incongruent as that used by Flourens, a method involving slicing through the entire cerebrum, would likely produce the types of results that Flourens in fact obtained, that is, an increasing loss of all functions.

The basic logic of the experimental method as developed by Flourens still permeates much contemporary research in physiological psychology and has two partially interdependent facets that deserve attention. The first supposes that the functions of the brain can be inferred by observing what behavioral alterations follow direct neurological manipulation. The second supposes that function can be localized at least in the sense of that part of the brain which was manipulated being a component of a larger system mediating the function underlying the behavioral alteration.

\section{INFERENCES FROM BEHAVIORAL ALTERATION}

The fundamental problem encountered in relation to the first facet is which categories of behavior or which categories of experience, or, in more classical terms. which faculties of the mind, are appropriate for characterizing behavioral alterations. Following the logic of Flourens, if we plan to observe what an animal ceases to do or begins to do after a brain ablation or during electrical stimulation of the brain, we must decide at some point what it is we are going to observe and under what conditions we are going to observe it. Is the decision to be based upon the philosopher's categories of perception. memory. volition. imagination. and so on? 
Do we instead resort to the sensory-motor categories that developed in the late 19th century and classify behavioral alterations in sensory and motor terms? Do we use categories which have in some sense biological significance for the organism? Encountered occasionally is the notion that such questions need not be of concern, for the functions of the brain will become evident, even obvious, in the nature of the alterations which follow brain lesions or which accompany electrical stimulation of the brain. However. as Teuber (1959) has emphasized in reference to the study of penetrating brain wounds in humans. changes in behavior are "often subtle, illusive, require very special tasks for their discovery, and even then might go undiscovered [p. 158]." A striking example of the subtle nature of the behavioral alteration that can follow major neurological insult is the notable absence of observable effects which follow sectioning of the corpus callosum. Only with the use of special testing procedures (Gazzaniga, Bogen, \& Sperry, 1962, 1963, $1965)$ does the role of the callosum in interhemispheric integration become evident. If special tests are required to detect behavioral change, then the importance of recognizing assumptions regarding functional categories and implicit conceptualizations concerning the organization of the nervous system becomes evident. Considerations such as the type of environment in which the organism is tested, the internal state of the organism at the time of testing, or the specific tasks employed to detect and measure behavioral change (and hence the manifestation of behavioral change), all depend upon the categories regarded by the $E$ to be relevant and significant. Whether an organism is tested, following some treatment, for changes in sensory thresholds, or on a perceptual task, or in a situation involving social interaction or in one involving the assessment of a motivational state depends upon these conceptualizations. If, for example, the organization and functions of the hypothalamus are conceptualized in terms of homeostatic regulatory mechanisms, the effects of manipulation of hypothalamic functioning will tend to be assessed in those terms and not in terms of changes in, for instance, memory or perceptual functioning (see Marshall, Turner, \& Teitelbaum, 1972). Hence, inferences about function based upon alterations in behavior produced by neurological manipulation will tend to be in terms of the original (explicit or more usually implicit) conceptualizations.

\section{FUNCTIONS OF THE BRAIN}

The logic of Flourens's experimental method of ablation called for observing the effects of removing a part of the nervous system. For Flourens, the parts of the nervous system were sixfold: the cerebrum, cerebellum, corpora quadrigemina, medulla, spinal cord, and spinal nerves. Young (1970) has suggested that this conception of the cerebrum's being a unitary structure was probably based largely on the philosophical influence of Descartes and the concept of the unity of mind. The ablation methodology adopted in the study of cerebral functioning, that involving surgical slicing through the entire cerebrum, of course was congruent with this conceptualization of the cerebrum, but, as noted earlier, this methodology would most likely generate results that would support the conceptualization even if it were incorrect.

The issue of just what constitutes a part of the brain is obviously of central importance for the question of localization of function. Should the hippocampus be considered as a unitary structure or can cytoarchitectonic differentiation be interpreted to indicate functional differentiation? Under what conditions can nuclei making up the amygdaloid complex be considered homogeneous and under what conditions must they be regarded as functionally distinct? Should two nuclei closely associated through a major fiber tract be considered a single part of the nervous system in terms of function? Should regions of the nervous system whose electrophysiological activity appear to covary be considered a single part? It is not the purpose of this paper to explore the issue of what constitutes a "part of the brain," but it is simply to be pointed out in passing that, given cerebral localization of function, an underinclusive conceptualization could lead to no detectable behavioral effects of some neurological manipulation if there is equipotentially. However, an overinclusive conceptualization may lead to bizarre and uninterpretable treatment effects and to the type of inference concerning localization of function made by Flourens.

It is basically neurological assumptions and conceptualizations of the type alluded to above which underlie one of the major criticisms of the study of brain-behavior relations through any form of direct manipulation of the brain. This stems from the brain's being regarded as functionally analogous to mechanical and, more recently, to electronic devices. The criticism is worthy of careful consideration, as it points clearly to how assumptions and conceptualizations can dictate methodology and as it points to the conditions under which it might be possible to, in some sense, localize function in specific brain structures.

The criticism can be exemplified with the argument of Gregory (1961), who has pointed out quite compellingly that there would be serious difficulty in attempting to deduce the functioning of an electronic circuit, such as a computer or television, by observing the effects on the circuit output of either "ablating" or removing component parts, or by "stimulating" the parts of the circuit with high-voltage probes. If a tube or a resistor is removed from a radio circuit and it is found that the radio begins to howl, it is unlikely that the function of the tube or resistor would be considered in terms of howling, that is, it is unlikely that it would be inferred that the normal function of the tube or resistor was to suppress or inhibit howling. Yet, as Gregory (1961) points out. this type of inference of some system's having the function of inhibiting some other 
svstem or some behavior is often encountered in physiological psychology. The reason for the difficulty is that in any complex serial processing circuit such as a radio or a television. the output is very dissimilar to the functioning of any of the component parts. The output of the system is described in terms different from those likely to be used to describe the function of the parts, and consequently it is unlikely that removing a part will indicate what the normal function of the part really is. Those who have paid for a television repairman need no reminding of the converse. that even when a circuit diagram is known and the principles of operation of each part are understood it can be very difficult to trace a malfunction given some abnormal output.

Part of the problem is that of distinguishing primary from secondary effects. In a complex circuit, seldom does the removal of a part produce an effect simply because the part is absent. Rather, the removel of the part disrupts the functioning of other components, and this induced malfunctioning itself produces some alteration in the output. Consequently, the alteration in the output of such a system might be due to the primary effects of the treatment and/or to secondary effects due to the alteration of the normal functioning of other parts of the system.

A second aspect of this problem is derived from the fact that, unlike most electronic circuits, biological organisms are adaptable and respond to changes in their behavior with other changes in their behavior. A minor facet of this is how to interpret transient behavioral change: has the organism adapted to the particular change, or was the change due to some psychologically trivial phenomenon such as brain edema? A second. and much more important. facet is concerned with the appearance of secondary effects which are an adaptation to or a response to the primary effects. In the context of brain ablations. it is possible to conceptualize the relationship between two behavioral alterations as being either independent or hierarchical. If they are related in a hierarchical manner. the organism shows a behavioral alteration. A. because of the existence of Behavioral Alteration B. a direct consequence of the lesion: or conversely. it shows B because of the existence of A. One could also imagine a double hierarchy of mutually reinforcing alterations. If the alterations are independent. they can be independent in one of at least two ways. Either the lesion or stimulation or other treatment produces the two alterations in behavior quite independently (as might be the case if there were a high degree of localization of function and the lesion invaded two different areas of the brain) or the two behavioral alterations are independent but are both secondary effects due to the treatment producing some underlying primary effect which causes both. Weiskrantz (1968b) has argued that it is possible. at least theoretically. to establish that behavioral alterations are independent within certain limits. He proposes that this can be done by producing Behavioral Alteration A by some means other than the original treatment and showing that Behavioral Alteration B does not occur, and conversely by showing that the induction of $B$ by some means other than the original treatment does not produce Behavioral Alteration A. But, he maintains, it is logically impossible to establish the existence of a hierarchical relationship, that is, to distinguish between primary and secondary effects.

These problems, among others, obviously raise serious questions about the utility of any form. of direct neurological manipulation in the study of brain-behavior relations. Gregory (1961) has, in fact, suggested that "to deduce the functioning of a part from the effect upon the output of removing or stimulating this part we must know at least in general terms how the machine works [p. 322]." Returning to the analogy of the television, he suggests that to interpret the effects of removing a part, it is necessary first to know the circuit diagram and, second, to understand the principles of operation of the circuit. Even as forceful an advocate of the use of direct manipulation of the brain as Weiskrantz (1968a) concedes that "we can make judgments about how some treatments affect the inner workings of the organism only if we already know something about the way they already work [p. 401] ]. However, Weiskrantz points out that the functioning of the brain is, in fact, not deduced in a logical manner, but that the neuroscientist works with sets of intuitive hunches and guesses and formulates hypotheses and models which he tests against reality and modifies. Furthermore, and more important for the present discussion, he argues that the brain is in fact not analogous to a television set or other electronic circuitry. First, the basic units of the nervous system, the neurons, are unlike electronic components in that they are digital and either fire or do not fire, and information presumably is encoded and processed partly in terms of the frequency and patternings of firing. However, since the processes which determine the digital output are the analogue processes of excitation and inhibition. Weiskrantz is much less skeptical of inferences about excitatory and inhibitory functioning based on dysfunction caused by ablation and stimulation than is Gregory. Indeed. he argues that this type of hypothesis has. in fact. been most fruitful in the past in generating research. A second reason for rejecting the electronic circuit analogy is that the mammalian nervous system is the product of multiple stages of evolution, with new sets of components and functional principles being superimposed upon previous sets, consequently maintaining the evolutionary history of the nervous system very much intact. In contrast, newer models of electronic devices are not the product of new components being added to old but often consist of entirely new types of integrated designs. In essence. then. Weiskrantz argues that because of its evolutionary development. the brain is not a serial information processing device made up of a large number of small and relatively independent processing units. 
How the organization of the nervous system is conceptualized, then, has enormous implications for methodology. Given that Gregory's analogy of the brain and electronic circuits is valid, that is, given that the brain acts as serial information processor in which information is taken in bit by bit and processed bit by bit by the entire circuit, his analysis of the utility of any direct neurological manipulation for the study of brain function would seem valid and well founded. However, given the orientation of Weiskrantz, that the brain acts as a parallel information processing device, one which consists of a large number of independent processing units, then even Gregory would probably concede the utility of methods like ablation and stimulation for the study of these independent units.

It is, then, no coincidence that those parts of the nervous system which, through the use of such methods as ablation and stimulation, are apparently best understood in functional terms at present are those which seem to act as parallel information processors, the sensory, motor, and certain homeostatic regulatory systems. Double dissociation can be easily demonstrated in the study of these systems. However, the problem with these methods is that, while they are appropriate for the analysis and dissociation of parallel processing systems, they are simply not tuned for the detection and study of the serial processing aspects of the nervous system, if there are such aspects.

\section{CONCLUSIONS}

It has been emphasized that the types of behavioral change looked for after neurological manipulation, as well as the methods of neurological manipulation themselves, are greatly influenced by how the functions of the brain and the structure of the brain are conceptualized. A comparison of the position represented by Gregory (1961) with that represented by Weiskrantz (1968a) further suggests that if the organization of the brain with respect to the processing of information relevant to some behavior is serial in nature, such methods as ablation and stimulation are unlikely to be of utility in unraveling questions of brain-behavior relations and functional localization; if it is paralled in nature, however, they may be. One of the important consequences of conceptualizing the nervous system as being a parallel information processing system and then proceeding to study it with methods like ablation and stimulation is that the results of such study will tend to support the validity of the conceptualization and will tend not to provide evidence against it. This is precisely the problem pointed out earlier in reference to the methodology of Flourens.

Flourens (1842), himself, said, "Everything in experimental research depends upon the method, for it is the method which gives the results [p. 502]." This paper has attempted to point out that method is very much determined by the assumptions and conceptualizations of the experimenter. It is these which determine the conditions under which the dependent variables are assessed and, indeed, the choice of the dependent variables themselves, and it is these which determine the choice of the independent variables. To the extent that method is dictated by assumptions and conceptualizations, then, everything (to overstate it somewhat) in experimental research depends upon assumptions and conceptualizations. It must be emphasized, though, that it is impossible to work without some set of assumptions, and this paper has attempted to make evident the importance of explicitly recognizing them and of recognizing the implications for methodology and data interpretation of working within one conceptual framework or another.

\section{REFERENCES}

Boring, E. G. A history of experimental psychology. (2nd ed.) New York: Appleton-Century-Crofts, 1950.

Ettlinger, E. G. (Ed.), Functions of the corpus callosum. London: Churchill, 1965.

Flourens, P. Researche expérimentales sur les proprietés et les fonctions du système nerveux dans les animaux vertébrés. (2nd ed.) Paris: Ballière, 1842.

Fried, P. A. Septum and behavior: A review. Psychological Bulletin, 1972, 78, 292-310.

Gazzaniga, M. S., Bogen, J. E., \& Sperry, R. W. Some functional effects of sectioning the cerebral commissures in man. Proceedings of the National Academy of Sciences, 1962, 48, 1765-1783.

Gazzaniga, M. S., Bogen, J. E., \& Sperry, R. W. Laterality effects in somesthesis following cerebral commissurotomy in man. Neuropsychologia, 1963, 1, 209-215.

Gazzaniga, M. S., Bogen, J. E., \& Sperry, R. W. Observations on visual perception after disconnexion of the cerebral hemispheres in man. Brain, 1965, 88, 221-236.

Goddard, G. V. Functions of the amygdala. Psychological Bulletin, 1964, 62, 89-109.

Gregory, R. L. The brain as an engineering problem. In W. H. Thorpe and O. L. Zangwill (Eds.), Current problems in animal behaviour. Cambridge: Cambridge University Press, 1961. Pp. 307-330.

Kimble, D. P. Hippocampus and internal inhibition. Psychological Bulletin, 1968, 70, 285-295.

Marshall, J. F., Turner, B. H., \& Teitelbaum, P. Sensory neglect produced by lateral hypothalamic damage. Science, 1971, 174, 523-525.

Olds, J. Approach-avoidance dissociation in rat brain. American Journal of Physiology, 1960, 199, 965-968.

Teuber, H.-L. Some alterations in behavior after cerebral lesions in man. In A. D. Bass (Ed.), Evolution of nervous control from primitive organisms to man. Washington, D.C: American Association for the Advancement of Science, 1959. Pp. 157-194.

Walker, A. E. The development of the concept of cerebral localization in the nineteenth century. Bulletin of the History of Medicine, 1957, 31, 99-121.

Weiskrantz, L. Treatments, inferences and brain function. In L. Weiskrantz (Ed,), Analysis of behavioral change. New York: Harper \& Row, 1968a. Pp. 400-414.

Weiskrantz, L. Some traps and pontifications. In L. Weiskrantz (Ed.), Analysis of behavioral change. New York: Harper \& Row, 1968b. Pp. 415-428.

Young, R. M. The functions of the brain: Gall to Ferrier (1808-1886). Isis, 1968, 59, 251-268.

Young, R. M. Mind, brain and adaptation in the nineteenth century. London: Oxford University Press, 1970.

(Received for publication July 31, 1973; accepted August 7, 1973.) 